\section{Prolapsing polyp and disappearing pseudocyst}

\author{
Gurminder S Mann FRCS Dileep N Lobo DM FRCS \\ Brian J Rowlands MD FRCS
}

J R Soc Med 2003;96:499-500

A gastric polyp, prolapsing into the duodenum, can result in acute pancreatitis.

\section{CASE HISTORY}

A woman of 70 was referred after six months of recurrent pancreatitis with $12 \mathrm{~kg}$ weight loss. She was otherwise well, was not on any regular medication and did not drink alcohol. Before transfer, a CT scan had been reported as showing a cystic mass in the head of the pancreas. Endoscopic retrograde cholangiopancreatography revealed a 'mucosal bulge' in the duodenum with normal delineation of the biliary tree. On repeat CT scanning the cystic mass was unchanged (Figure 1a). The pancreas was oedematous. When her pancreatitis recurred, with raised serum amylase, alkaline phosphatase, alanine aminotransferase, gammaglutamyl transferase and bilirubin, a further CT scan revealed, in addition to the cystic mass, dilatation of the extrahepatic and intrahepatic biliary ducts. Laparotomy was decided on with the aim of draining the cyst and performing a cholecystectomy. At operation the gallbladder was seen to be dilated, with a swelling behind the first part of the duodenum which promptly disappeared after decompression of the common bile duct. After routine cholecystectomy the common bile duct was explored with a choledochoscope. No stones were identified.

Her recovery was uncomplicated until postoperative day 21 when the symptoms of pancreatitis recurred, with increases in serum amylase, alkaline phosphatase, alanine aminotransferase, and gamma glutamyl transferase but normal bilirubin. A CT scan now showed a thick walled cystic lesion superior to the tail of the pancreas pushing into the lesser curvature of the stomach (Figure $1 b$ ). There was no cyst in the head of the pancreas. On gastroscopy a large mobile pedunculated polyp was seen in the fundus of the stomach, and a barium meal showed that the polyp was prolapsing into the duodenum (Figure 2). At repeat

Section of Surgery, University Hospital, Queen's Medical Centre, Nottingham NG7 2UH, UK

Correspondence to: Mr D N Lobo, Section of Surgery, E Floor, West Block, University Hospital, Queen's Medical Centre, Nottingham NG7 2UH, UK

E-mail: dileep.lobo@nottingham.ac.uk

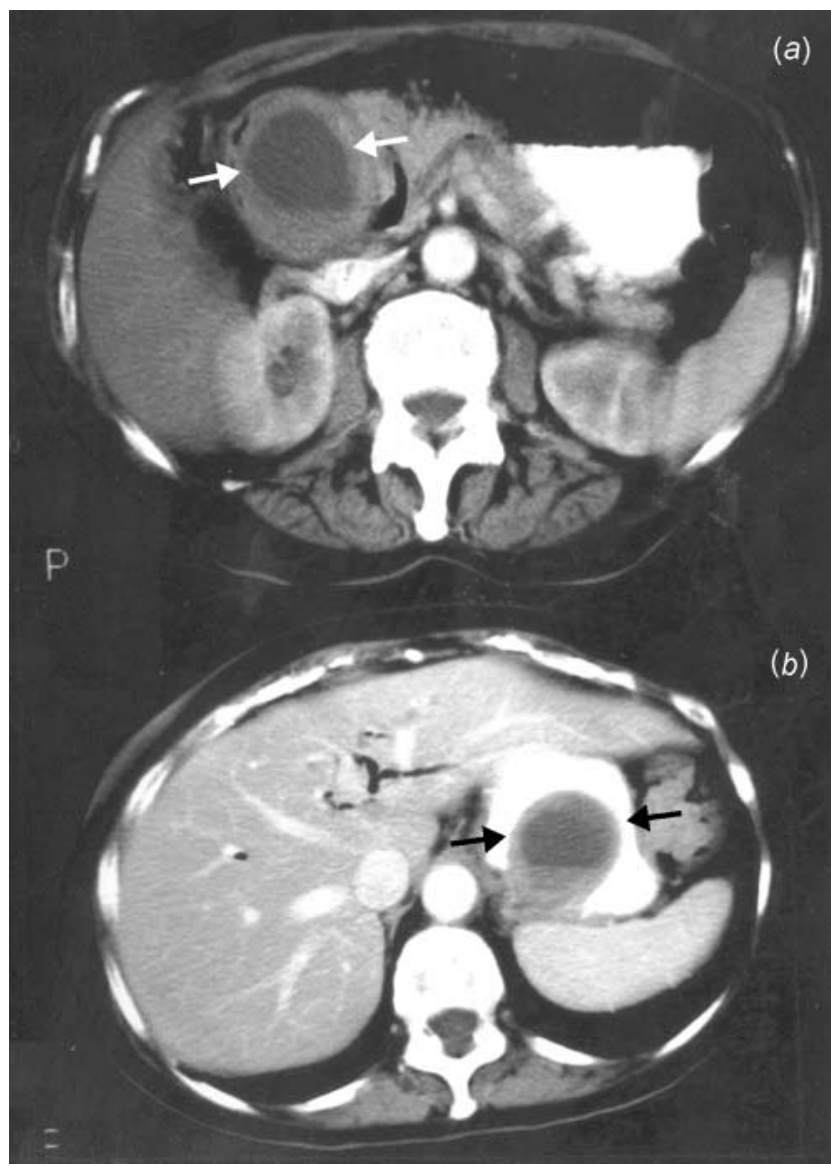

Figure 1 Contrast enhanced abdominal CT scans. (a) White arrows show cystic lesion in the region of the pancreatic head. (b) Subsequent scan shows this lesion within stomach (black arrows)

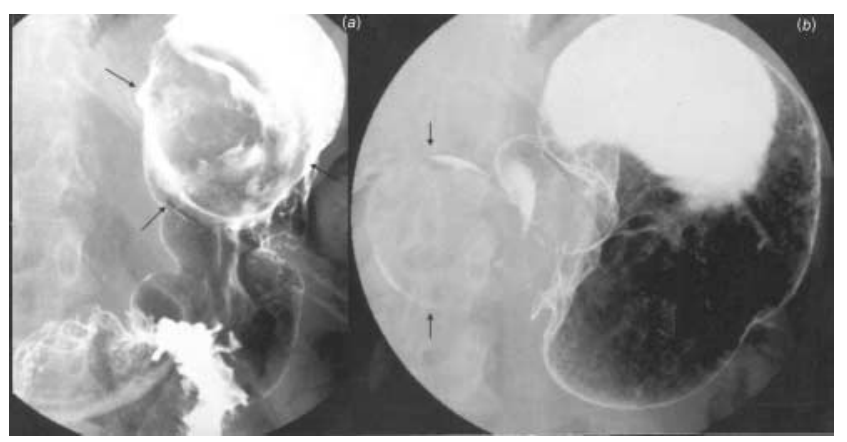

Figure 2 Double-contrast barium meal. Large polyp (arrows) arising from the gastric fundus (a) and prolapsing into the duodenum (b)

laparotomy the polyp, which was about $5 \mathrm{~cm}$ in diameter, was delivered and excised via a gastrotomy. It proved to be a benign cystic leiomyoma. Eighteen months later there had been no symptom recurrence and the lost weight had been regained.

\section{COMMENT}

Exclusion of gallstones, alcohol and drug intake in a patient with acute pancreatitis often prompts a diagnosis of 
idiopathic pancreatitis. We have found only three reports ${ }^{1-3}$ on pancreatitis associated with a gastric polyp and none with repeated episodes as in the present case.

Gastroduodenal intussusception is most commonly due to a benign tumour, ${ }^{1}$ and Gardner's syndrome (familial adenomatous polyposis) ${ }^{2}$ is one condition in which it has caused pancreatititis. ${ }^{2}$

A cystic lesion in the vicinity of the pancreas on CT scan, in a patient with a history of acute pancreatitis, is usually interpreted as pseudocyst. In the patient reported here this misinterpretation led to a delay in diagnosis and definitive treatment.

\section{REFERENCES}

1 Kleinhaus U, Weich YL, Maoz S. Gastroduodenal intussusception secondary to prolapsing gastric tumours. Gastrointest Radiol 1986;11:229-32

2 Herman LL, Kurtz RC, Brennan MF, Shike M. Acute pancreatitis from intussusception of a gastric polyp in a patient with Gardner's syndrome. Dig Dis Sci 1992;37:955-60

3 White PG, Adams H, Sue-Ling HM, Webster DJ. Case report: gastroduodenal intussusception - an unusual cause of pancreatitis. Clin Radiol 1991;44:357-8

\section{Disseminated herpes simplex after total skin electron beam radiotherapy for mycosis fungoides}

\author{
Benjamin D Smith MD Chang Bae Son MD ${ }^{1}$ \\ Lynn D Wilson MD MPH
}

$J$ R Soc Med 2003;96:500-501

In mycosis fungoides, CD4+ T-cells localize to the skin, resulting in patches, plaques, tumours, and erythroderma. Superficial radiotherapy delivered with electrons to all skin surfaces is an important component of treatment in this disease. A theoretical risk of total skin radiotherapy is cutaneous immunosuppression.

\section{CASE HISTORY}

At age 76, a woman with a history of chronic lymphocytic leukaemia, hypothyroidism, recurrent herpes zoster, and cutaneous basal and squamous cell carcinomas developed

Departments of Therapeutic Radiology and ${ }^{1}$ Dermatology, Yale University School of Medicine, New Haven, CT, USA

Correspondence to: Lynn D Wilson MD, Yale University School of Medicine, Department of Therapeutic Radiology, 333 Cedar Street, HRT 136, New Haven, CT 06520, USA

E-mail: lynn.wilson@yale.edu mycosis fungoides involving the trunk and right shoulder. Despite treatment with multiple systemic and topical agents, the disease progressed and 4 years later she was referred to a radiation oncologist. At that time she had circular, deep pink, 1-6 $\mathrm{cm}$ patches and plaques on more than $10 \%$ of her body surface area localized primarily to the trunk, arms, and legs and with ulceration in the gluteal cleft and perineal region. There was no obvious adenopathy or hepatosplenomegaly.

Total skin radiotherapy was initiated with 6 megaelectronvolt electrons produced by a linear accelerator mounted on a dual-angle gantry. The patient was treated with 100 centigray (cGy) per day, 4 days per week, by a standard six-field technique. ${ }^{1}$ After receiving 500 cGy she became severely fatigued and sought medical attention. On examination she had developed numerous $2-3 \mathrm{~mm}$ vesicles, pustules, yellow-crusted erosions, and haemorrhagic erosions, all of which were localized to the previously described plaques (Figure 1). A Tzanck smear gave an equivocal result but a direct immunofluorescence test for herpes simplex virus was positive. She was admitted to hospital and treated with intravenous aciclovir, $10 \mathrm{mg} / \mathrm{kg}$ every 8 hours for 24 hours then $5 \mathrm{mg} / \mathrm{kg}$. By hospital day five, all of her lesions had encrusted. She was discharged on oral valaciclovir $1 \mathrm{~g}$ twice daily and has not resumed radiotherapy.

\section{COMMENT}

This patient developed Kaposi's varicelliform eruption (KVE), a disseminated vesicular eruption localized to plaques from a pre-existing lesion. KVE most frequently occurs in patients with atopic dermatitis, but has been reported in other skin diseases including mycosis fungoides. $^{2-4}$ Herpes simplex virus is the primary cause, but individuals with atopic dermatitis who receive the smallpox (variola) vaccine may develop an identical clinical syndrome.

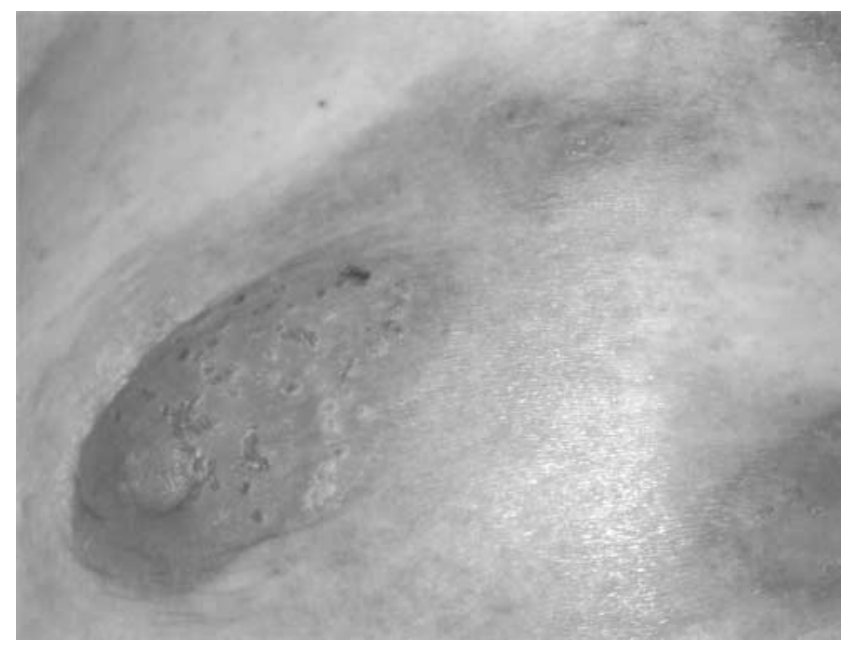

Figure 1 A cluster of vesicles and erosions arising on a pre-existing cutaneous plaque 